\title{
PANDEMIA Y TURISMO
}

\section{EL TURISMO LATINOAMERICANO FRENTE A LA CRISIS DEL CORONAVIRUS}

\author{
Lyudmila P. Voronkova
}

Doctora titular (Filosofia), prof., (voronkova@fgp.msu.ru)

Jefa de la Cátedra de Comunicaciones Globales

Facultad de Estudios Globales

Universidad Estatal LOMONÓSOV de Moscú

Léninskiye gory, 1, Moscú, 119991, Federación de Rusia

Recibido el 22 de abril de 2021

Aceptado el 7 de julio de 2021

DOI: $10.37656 / \mathrm{s} 20768400-2021-4-07$

Resumen. En el presente artículo son examinados los procesos que se observan en el turismo latinoamericano bajo la influencia de la crisis del coronavirus. El objetivo de este trabajo es el de revisar los efectos de la pandemia en la industria del turismo en los países de América Latina y el Caribe y, las orientaciones que se proponen para su recuperación. Sobre la base de datos estadísticos, presentados en publicaciones de la UNWTO (Organización Mundial del Turismo), del portal global Statista de datos de negocio y de otros análisis son exhibidas las particularidades de la reorganización que tiene lugar en el turismo latinoamericano. Los expertos ven la salida de la situación creada en la orientación a la digitalización del sector del turismo, en la búsqueda de nuevos tipos de descanso para los consumidores que trabajan a distancia $y$, en otras innovaciones. En este estudio se destaca que, a pesar de las distintas estrategias gubernamentales de combate a la pandemia, para el relanzamiento de toda la industria del turismo es importante la cooperación multilateral de todos los países de América Latina y el Caribe en la tarea destinada a remontar las consecuencias de la crisis del coronavirus.

Palabras clave: turismo latinoamericano, crisis del coronavirus, turismo dentro del continente, concepción negocio más ocio, concepción Hotel Oficina, marketing digital, ventas online, turismo "post pandemia" 
El turismo latinoamericano frente a la crisis del coronavirus

\title{
LATIN AMERICAN TOURISM IN FACE OF THE CORONAVIRUS CRISIS
}

\author{
Lyudmila P. Voronkova \\ Dr. Sci. (Philosophy),prof., (voronkova@fgp.msu.ru) \\ HoD of Global Communications \\ Faculty of Global Studies \\ LOMONOSOV Moscow State University \\ 1, Leninskie gory, Moscow, 119991, Russian Federation
}

Received on April 22, 2021

Accepted on July 7, 2021

DOI: $10.37656 / \mathrm{s} 20768400-2021-4-07$

Abstract. The article examines the processes taking place in Latin American tourism under the influence of the coronavirus crisis. The purpose of this article is to review the impact of the pandemic on the tourism industry in Latin America and the Caribbean and proposed directions for its recovery. Based on statistical data presented in the publications of the World Tourism Organization (UNWTO), the global business data platform Statista and other analytical materials, the features of the ongoing reorganizations in Latin American tourism are shown. Experts and specialists see a way out of this situation in the orientation towards digitalization of the tourism industry, the search for new types of tourism for consumers working remotely and other innovations. The article notes that despite various government strategies to combat the pandemic, multilateral cooperation of the LAC countries in overcoming the consequences of the coronavirus crisis is important for restarting the entire tourism industry

Keywords: Latin American tourism, coronavirus crisis, inland tourism, Business pleasure concept, Hotel Office concept. digital marketing, online sales, post-pandemic tourism

\section{ЛАТИНОАМЕРИКАНСКИЙ ТУРИЗМ ПЕРЕД ЛИЦОМ КОРОНАВИРУСНОГО КРИЗИСА}

Людмила Петровна Воронкова

Д-р филос. наук, проф., (voronkova@fgp.msu.ru)

Зав. кафедрой глобальных коммуникаиий

Факультет глобальных процессов

Iberoamérica, 2021, núm. 4, pp. 146-164 
Lyudmila P. Voronkova

МГУ имени М.В. Ломоносова

РФ, 119991, г. Москва, Ленинские горы, д. 1

Статья получена 22 апреля 2021 г.

Статья принята 7 июля 2021 г.

DOI: $10.37656 / \mathrm{s} 20768400-2021-4-07$

Аннотация. В статье рассмотрены процессы, происходящие в латиноамериканском туризме под влиянием коронавирусного кризиса. Цель настоящей статьи - рассмотреть воздействие пандемии на индустрию туризма в странах Латинской Америки и Карибского бассейна и предлагаемые направления ее восстановления. На основе статистических данных, представленных в публикациях Всемирной туристской организаџии (ЮНВТО), глобальной платформы бизнесданных Statista u других аналитических материалах, показаны особенности происходящих реорганизачий в латиноамериканском туризме. Выход из создавшегося положения эксперты и специилисты видят в ориентациии на изифровизацию туристской отрасли, поиск новых видов туризма для потребителей, работающих удаленно и других инновачиях. В статье отмечается, что несмотря на различные правительственные стратегии борьбы с пандемией для перезапуска всей индустрии туризма важно многостороннее сотрудничество стран ЛАК в преодолении последствий коронавирусного кризиса.

Ключевые слова: латиноамериканский туризм, коронавирусный кризис, внутриконтинентальный туризм, конщепџия Business pleasure, концегпия Hotel Office, uиифровой маркетинг, опline-продажи, «постпандемический» туризм

La crisis del coronavirus dio a principios del siglo un vuelco radical a nuestro mundo colapsando la economía global. El turismo internacional fue el sector más azotado por ella. La industria del turismo ha sufrido prácticamente en todos los países los impactos más graves resultando arrojada al nivel de comienzos de los 90 del siglo pasado. Los gobiernos de América Latina y de la cuenca del Caribe, al igual que la mayoría de los países enfrentaron el COVID-19 que ha desatado, a juicio de los investigadores "la segunda Gran depresión o Gran cuarentena", cuya profundidad habrá que calibrar todavía [1]. Ello no 
obstante que, en los países de la región, el turismo anterior a esta pandemia exhibía buenos resultados. En el Índice de capacidad competitiva de viajes y de turismo, del Foro Económico Mundial (WEF) se constataba que, 12 de 21 países de América Latina y el Caribe habían elevado su competitividad, en el periodo de 2017 a 2019, incluyendo naciones tales como Bolivia, Colombia, Uruguay y República Dominicana [2]. México es uno de los protagonistas clave del sector turístico a escala internacional. En 2019, México batió el récord en número de turistas extranjeros acogidos, de más de 45 millones, prácticamente un $9 \%$ más del año anterior. El Estado azteca fue el único país de la región integrante de la lista estelar de los 10 más visitados del mundo [3].

La situación del turismo se muestra heterogénea en las condiciones presentes en distintos países de América Latina y el Caribe, dando origen en tanto a una imagen bastante abigarrada de las consecuencias de la pandemia y de las medidas tomadas por los gobiernos contra ella. A medida que han comenzado a conocerse informaciones sobre la atenuación del ritmo de la propagación del COVID-19, los especialistas se han dado a pensar en cómo sacar el sector del turismo de su crisis profunda. En la prensa aparecieron variantes distintas de resurgimiento y desarrollo del turismo postpandémico. Este artículo se plantea revisar la influencia de la pandemia en la industria de turismo en los países de América Latina y el Caribe y las orientaciones que se proponen para su recuperación En qué consisten las particularidades de la crisis del coronavirus y, cuáles son las posibles vías de salida de la crisis en los países de América Latina y del Caribe, son las interrogantes que están en el centro de la atención de científicos, políticos y de la opinión pública de estos países. 
El tema de la crisis del coronavirus en el turismo ha comenzado a estudiarse activamente en los últimos tiempos. Entre los trabajos de especialistas rusos, dedicados a las cuestiones generales de la influencia de la pandemia, valga poner de relieve las publicaciones de I.V. Loguntzova, A.V. Budaiev, S.A. Butórov, E.V. Maxímova, V.V. Morózov, S.S. Soldátova, S.M. Anpílov, N.A. Shkoliar, L.S. Ókunieva, I.Y. Shwetz. Los problemas del impacto del COVID-19 en el sector del turismo son examinados en el artículo de P.A. Kuchínov, quien destaca que el proceso gatillado conduce a cambios profundos y a la generación de un nuevo orden mundial en esta esfera [4].

En cuanto a los investigadores extranjeros valga mencionar al científico mexicano Werner Alejandro, a los investigadores estadounidenses Edward B. Barbier, a Joanne C. Burgess, al vicepresidente ejecutivo de JLL Hotel and Hospitality Dan Fenton, al científico venezolano Marino J. González, al investigador bélgico Noel B. Salazar.

En el artículo presentado la nota conceptual del secretario general de la ONU conformó la base empírica y estadística sobre el turismo y el COVID-19, amén de otros textos informativoanalíticos de la ONU. En este trabajo se recurrió a informes de la Organización Mundial de Turismo (OMT) de la ONU [United Nations World Tourism Organization (UNWTO)], del Fondo Monetario Internacional (FMI), del Consejo Mundial de Viajes y Turismo [The World Travel \& Tourism Council (WTTC)], de la Comisión Económica de la ONU para América Latina y el Caribe (CEPAL) [The Economic Commission for Latin America (ECLA)], informe sectorial de la Organización Internacional del Trabajo (OIT) sobre el turismo, además de materiales de sitios web, especializados en la exposición del estado del turismo en 
países de América Latina y el Caribe. A todo esto fueron sumados otros trabajos contenidos en publicaciones del Instituto de Latinoamérica de la Academia de Ciencias de Rusia, así como en revistas especializadas en el negocio turístico.

Las olas de la pandemia se abalanzaron prácticamente en último lugar sobre el subcontinente latinoamericano. A pesar de ello, los países de la región no supieron aprovechar el receso registrado en mayo de 2020. Y América Latina iba a ser declarado el nuevo epicentro del coronavirus, en el que se concentró más del $40 \%$ de los casos de muertes en el mundo. La epidemia, como apuntaban científicos, afectó en un comienzo a la población pudiente que retornaba de balnearios extranjeros, europeos, para a continuación extenderse rápidamente entre grupos de la población de bajos ingresos, los que prácticamente no tenían acceso a los servicios médicos [5].

La crisis provocada por la pandemia de COVID-19 ha sido una dura prueba para los países de América Latina y el Caribe. El bajo nivel de la demanda turística, a raíz de las rígidas medidas impuestas de contención, de distanciamiento social, la renuncia de los turistas a los viajes, a causa de los temores crecientes, llevaron a que el año 2020 fuese el peor para el sector del turismo de la región. Los expertos en turismo, al referirse al impacto del COVID-19 en este sector en 2020 ponen de relieve su carácter sistémico. Los cambios alcanzaron tanto los flujos turísticos como la esfera del empleo en el sector mismo. Según estimaciones de la CEPAL, en los primeros cuatro meses de 2020, el número de turistas que viajaban a América del Sur y Central se redujo en un $35 \%$ y, a la cuenca del Caribe, en un 39\% [6].

Como consecuencia de ello, en países latinoamericanos y caribeños crecieron el desempleo y la tensión social. En la citada 
Nota conceptual del Secretario general de la ONU se apuntaba que en agosto de 2020, el desempleo amenazaba a " 100 millones de puestos laborales directos en el turismo, aparte de las plazas en sectores vinculados a este, tales como las ramas que demandan uso intensivo de mano de obra, como el alojamiento y el catering, que proporcionan empleo a 144 millones de trabajadores en todo el mundo" [7]. En el segundo trimestre de 2020, Brasil, Chile, Colombia, México y Perú perdieron un total 30 millones de empleos, resultando singularmente afectados las mujeres, los jóvenes y trabajadores de bajo nivel de educación [8]. Comenzaron a sentirse con agudeza los problemas de seguridad ecológica y biológica en los destinos turísticos habituales.

La pandemia del COVID-19 tuvo para los países de la región consecuencias políticas y socioeconómicas negativas puesto que, condujo al incremento de los ánimos de protesta también de los antiglobalistas. Los investigadores plantean a menudo la pregunta de por qué no pudieron los países de la región prepararse oportuna y debidamente para mitigar las consecuencias o, incluso, frenar la propagación del virus del COVID-19 y, si era posible evitar en general sus estragos. Por el contrario, con excepciones muy escasas, los países de la región se vieron de cara a la pandemia, prácticamente inermes.

La pandemia se superpuso a la situación específica del turismo en esta región, que está asociada con la situación geopolítica desfavorable en varios países, con la distribución desigual de los recursos turísticos entre los países y, también, con las particularidades y las orientaciones de los torrentes de turismo, amén de otras. Además, la mayoría de los países latinoamericanos se desarrollaban de acuerdo con modelos vinculados a las graves restricciones estructurales en la 
economía que condujeron, a juicio de especialistas, al surgimiento de vulnerabilidades sistémicas frágiles, manifestadas en distintas esferas de la sociedad.

La infraestructura atrasada de la salud pública $\mathrm{y}$, prácticamente, la ausencia total de la protección social de la población en una serie de países de la región es presentada, con frecuencia, como una de tales vulnerabilidades. Según estimaciones de la Organización Panamericana de la Salud (OPS), la desfavorable situación en la sanidad se debe a las inversiones estatales insuficientes. $E$ incluso el incremento de los gastos fiscales en la salud pública en un $6 \%$ es, según OPS, "indispensable, aunque condición insuficiente para reducir las desigualdades de la cobertura sanitaria general" [9].

Como segunda causa de la indefensión de la población se mencionan las medidas no coordinadas de los gobiernos de algunos países. En los medios de comunicación se cita a menudo lo expresado por el presidente de Brasil Jair Messias Bolsonaro quien, hablando para la prensa declaró que "los brasileños no se contagian con nada", aunque a la sazón, en el país, solo según las informaciones oficiales, había ya unos 3 mil contagiados, situándose como el Estado líder del continente por el número de víctimas de la pandemia [10].

Los gobiernos latinoamericanos han venido optando por estrategias distintas de combate al coronavirus. Muchos países latinoamericanos y caribeños adoptaron operativamente medidas sanitarias e implantaron el distanciamiento social. Argentina fue, en este sentido, uno de los más operativos, al introducir a partir del 19 de marzo de 2020 la cuarentena obligatoria total para todos. Otros países se limitaron a proscripciones parciales o, adoptaron otras medidas orientadas mucho más a la mitigación de las consecuencias de la crisis. Ello hizo que la situación en la 
región deviniera más complicada y ambigua. La Organización para la Cooperación y el Desarrollo Económicos (OCDE) presentó un resumen de la reacción de los gobiernos de 15 países que forman parte del Grupo dirigente del programa regional de la OCDE para América Latina y el Caribe [11].

El presidente de México Andrés Manuel López Obrador anunció, a principios de diciembre de 2019, que se pronunciaba en contra de la introducción de medidas restrictivas severas para combatir la propagación del coronavirus COVID-19. Como resultado fue limitada parcialmente la circulación de ciudadanos, además de la implantación de algunas prohibiciones en el terreno de la economía, de la educación y de la salud pública [12]. A partir de enero, en cada Estado del país rigen medidas propias de seguridad. En el país está en marcha la vacunación. Así, según fuentes del portal global Statista, por la cantidad de dosis de vacunas contra el COVID-19, inyectadas al 24 de marzo de 2021, México se encontraba en el décimo octavo lugar en el mundo (5.926.967). Entre los países del subcontinente, está aventajado solo por Brasil, que se encuentra en el sexto lugar con 17.477. 952 dosis inyectadas [13]. Aparte de esto, la innovación más sobresaliente de la dirigencia mexicana fue el proyecto informativo denominado semáforo federal de cuatro colores, por el que, las zonas de color rojo, naranja, amarillo y verde advierten a turistas y a los ciudadanos de la situación epidemiológica [14].

Actualmente está claro que, para los países latinoamericanos y caribeños que han elegido estrategias distintas para combatir las consecuencias de la pandemia ha resultado difícil enfrentar la crisis del coronavirus y sus secuelas. En el informe del Banco Mundial sobre América Latina y el Caribe titulado "La economía en tiempos del COVID-19" se indicaba que, los países 
de ALC están empeñados en frenar la propagación de la epidemia, no permitiendo al mismo tiempo un receso brusco de la actividad económica. En el documento son analizadas las medidas de los gobiernos que "tratan de encontrar un compromiso entre la pérdida de vidas y las pérdidas económicas, aunque esto pueda parecer incluso reñido con la ética" [15]. Los expertos del Banco Mundial estiman que, los países de la región tendrán que encontrar un equilibrio entre gastos potenciales en la salud de la ciudadanía y la eficacia de las estrategias acometidas de contención de la epidemia, a fin de conjurar el colapso del sector financiero.

Uno de los pasos en esta dirección puede ser considerado el deseo de trazar un plan de la "Nueva realidad". Y se subraya que ello no entraña el retorno al estado del turismo existente antes de la pandemia, o como apuntan los científicos, no es el retorno a la "normalidad" que existía anteriormente, sino una reconsideración total del futuro [16].

Están apareciendo cada vez más publicaciones dedicadas a la búsqueda de mecanismos innovadores de la política estatal para alcanzar los objetivos de un desarrollo sostenible sobre una base económicamente eficaz [17]. Los gobiernos de los países, a la hora de adoptar estrategias para la revitalización y el desarrollo de la industria del turismo han optado por la vía de atenuar, o incluso de levantar las medidas de cuarentena. Esta postura es apoyada por los expertos de la Organización Mundial de Turismo, que consideran que las restricciones de los viajes son un escollo para el restablecimiento del turismo internacional. Además, subrayan que los gobiernos deberían abordar de manera más integral el problema de la recuperación del turismo.

En el informe de la ONU "COVID-19 y la transformación del turismo" fue presentada la Hoja de ruta sobre la 
reconstitución del sector del turismo, en la que se destaca que su resurgimiento es imposible sin el restablecimiento de toda la economía, sin el estímulo de la capacidad competitiva y la elevación de su vitalidad, sin la promoción de un entorno empresarial para las micro, pequeñas y medianas empresas. La Hoja de ruta prevé, además, la gestión de la crisis y la mitigación de las secuelas socioeconómicas y también el fortalecimiento de la coordinación, de las relaciones de cooperación y de la solidaridad con miras a la recuperación económica [18].

Los lineamientos prioritarios de la reconstrucción de sector turístico en los países de ALC deben ser alineados considerando las singularidades de esta región. Para ello es necesario pensar en las orientaciones correctas y, a juicio de los especialistas, uno de tales direcciones puede ser el turismo interno. Para los países de ALC habitualmente, el grueso de los turistas lo conformaban habitantes de América del Norte. Tomando en cuenta que, EE.UU., Canadá con los países de América Central, sobre todo con México, conformaban los principales flujos intracontinentales de turistas, muchos Estados latinoamericanos y caribeños adoptaron medidas para su estímulo. Así, según la Organización Mundial del Turismo, México figura en el quinteto de países a los que norteamericanos y canadienses están dispuestos a viajar, en primer lugar, una vez terminada la pandemia. Decenas de miles de turistas norteamericanos, a fin de escapar del invierno pandémico en América del Norte arribaron, en los inicios del 2021, a las playas del litoral caribeño de México [19].

A la recuperación del turismo va a contribuir también la elaboración de una nueva estrategia de trabajo con clientes potenciales, o sea, turistas. La pandemia cambió en buena 
medida la psicología y la conducta de los turistas. Los especialistas subrayan que es indispensable recuperar la seguridad de los viajeros e incentivar la demanda turística. Con tal fin se llevan a cabo investigaciones para estudiar la reacción de los viajeros frente a riesgos, como las consecuencias ante posibles problemas de salud durante un viaje.

Uno de los efectos de la pandemia ha sido el surgimiento y la formación de un nuevo tipo de turistas. En 2020, según observaciones de agentes de turismo, creció la demanda de una nueva pauta de descanso, el bleisure, o business +leisure, o sea, trabajo a distancia sin interrumpir el descanso. Entre los turistas abundan los empleados de compañías que trabajan a distancia, razón por lo que pueden viajar y descansar simultáneamente. Para este nuevo contingente de turistas, están siendo creados programas enmarcados en la concepción de negocio y placer (Business pleasure) con nuevos requisitos para los hoteles, dentro del espíritu de la concepción del Hotel Oficina. El programa comprende las comodidades del hotel y la posibilidad de ocuparse de asuntos de rabajo, ofreciendo para ello a los clientes los servicios propios de una oficina con Wi-Fi estables de alta velocidad. Esta concepción ha sido introducida con excelencia por Accor Hotels, el primer operador mundial francés en el sector de viajes y de turismo, que cuenta en ALCaribe con más de 200 hoteles [20].

Expertos de la Organización Mundial de Turismo, estiman que es indispensable repensar completamente un modelo de negocio que restablezca el turismo sobre la base de la digitalización y de la aplicación de tecnologías innovadoras. La digitalización, de la que se viene hablando hace ya tiempo, ha comenzado a ser considerada el eje magistral del turismo postCOVID. La ONU instaba no hace mucho a la recuperación 
post pandémica social y económica de los países latinoamericanos y caribeños mediante la "transformación digital". En este plano es elocuente la notable feria Tianguis Turístico Digital México 2021, inaugurada el 23 de marzo de 2021. Habiendo sido suspendida en 2020 partió en este año en formato digital, animada por el lema, "La época digital es la llave para el turismo". Los países de América Latina y el Caribe disponen de los fundamentos suficientes para la aceleración de los procesos de digitalización de la esfera del turismo. En 2019, más del $67 \%$ de la población de ALC tenía acceso a Internet. Sus líderes son Brasil, Argentina, Chile, Colombia, Nicaragua, Perú y Venezuela. Aunque, los especialistas apuntan que la situación en los distintos países es muy heterogénea en este sentido [21].

Ello contribuyó, en las condiciones de la pandemia, a un desarrollo rápido de la comercialización digital, orientada a la captación de clientes potenciales en el punto geográfico que se encuentren. El desarrollo del mercado digital, la creación de los canales eficaces de promoción de los destinos turísticos coadyuvaron al incremento de las compras remotas, a la irrupción del comercio electrónico entre los consumidores de servicios turísticos.

El sondeo de opinión efectuado por el EBANX, una cotizada institución de pagos en América Latina, revelaba que, en el año en curso, el comercio electrónico en la región va a crecer un $8,49 \%$, e incluso, no obstante la caída de la demanda de viajes, puede llegar a ser un mercado de más rápido crecimiento en el mundo [22]. Si en 2020, el número de usuarios de comercio electrónico rebasaba los 267 millones, se estima que para 2024, esta cifra crecerá un 31\%, llegando a cerca de los 351 millones de usuarios en toda la región. El mercado digital de América 
Latina tiene ciertas perspectivas, debido a que el $78 \%$ de los compradores digitales manifestaron el deseo de continuar, también después de la pandemia, los contactos de compra en formato digital [23]. Es más, en algunos países como Argentina, este índice podría llegar al 92\% [24].

La situación actual evidencia que es necesario considerar el turismo latinoamericano a través del prisma de, antes y después de la pandemia del COVID-19. Antes de la pandemia, el FMI calculaba para dicha región un crecimiento económico de un poco más del 1,5\%. La crisis del coronavirus en 2020 condujo al desplome del PIB en más de un 7\%, y en 2021, el FMI corrigió la baja hasta un 5,8\% [25]. Es indudable que la pandemia puso al desnudo todas las zonas problemáticas de las economías de los países de América Latina y el Caribe, y mostró un sinnúmero de deficiencias estructurales en los sistemas políticos y sociales, entre ellas, la descoordinación de la actividad de las organizaciones regionales internacionales en el combate al virus.

La búsqueda de las vías de recuperación del sector del turismo debe considerar que, por una parte, resulta sumamente difícil predecir la fecha del fin de la pandemia. Los elevados índices de contagio y de mortandad retrasan los procesos de restablecimiento del sector turístico en los países latinoamericanos y caribeños. Brasil es el país del subcontinente más azotado a causa de la pandemia del COVID-19. En el país se habían registrado hacia abril de 2021 unos 14 millones 300 mil casos confirmados de COVID-19. Y en general, en la región se cuentan más de 28 millones de pacientes diagnosticados [26].

Expertos de la Organización Mundial de Turismo confíaban, dentro de los distintos escenarios para el turismo, en que su recuperación a nivel internacional comience hacia el tercer trimestre de 2021. Mientras que el 20\% de los especialistas 
estiman que ello sobrevendrá no antes de 2022 y, que el retorno al nivel de 2019 podría tomar de dos años y medio a cuatro [27], lo que en parte se debe al segundo brote global, de principios de 2021, del COVID-19.

De otra parte, a la hora de planear las medidas para la recuperación del turismo es importante considerar que ha cambiado la situación y el enfoque con respecto a la vida en general. Las comunicaciones a distancia están abriendo ante el turismo nuevos horizontes y nuevos formatos de colaboración internacional.

Una vulnerabilidad clave de los países latinoamericanos y caribeños consiste en la falta de mecanismos integrales de paliación de las consecuencias socioeconómicas de la pandemia, lo que ha evidenciado hasta qué punto está interconectado el mundo contemporáneo $\mathrm{y}$, que los problemas de la seguridad de cada uno están vinculados a la seguridad de todos [28]. Se puede considerar un problema global la superación de las consecuencias de la crisis del coronavirus, debido a que no pueden ser resueltas con los esfuerzos de un solo país. El secretario general de la Organización Mundial del Turismo Zurab Pololikashvili y el secretario general de la OCDE, Ángel Gurria subrayaban que son de suma importancia, en interés de la recuperación segura y estable del turismo, "la cooperación multilateral reforzada y el apoyo pleno. La colaboración y la coherencia en la regulación de los viajes, a nivel bilateral, regional e internacional son los medios que ayudarán a relanzar el turismo seguro, acelerarán el restablecimiento de la economía e infundirán esperanzas en millones de personas [29]. Ello va a adquirir relevancia especial en los países de América Latina y el Caribe, debido a que ayudaría a robustecer la confianza con respecto a la capacidad de los gobiernos de los países de la 
región a crear un nuevo modelo de desarrollo, elaborándose para ello una Hoja de Ruta asentada en los derechos humanos y, orientada a alcanzar un desarrollo estable digno.

\section{Bibliografía References Библиография}

1. Lanchimba Cintya, Bonilla-Bolaños Andrea, Díaz-Sánchez Juan Pablo. The COVID-19 pandemic: theoretical scenarios of its socioeconomic impacts in Latin America and the Caribbean. Brazil. J. Polit. Econ. vol.40 no.4 São Paulo Oct./Dec. 2020. Epub Oct 16, 2020. Available at: http://www.scielo.br/scielo.php?script=sci_arttext\&pid=S0101 31572020000400622 (accessed 07.07.2021).

2. Fenton Dan. These 6 strategies can help tourism recover in Latin America, 15 Oct. 2020. Available at: https://www.weforum.org/agenda/2020/10/these-6-strategies-can-helptourism-recover-in-latin-america-df689974cd/ (accessed 17.07.2021).

3. Самые посещаемые страны мира в 2019 году. 15.02.2020 [The most visited countries in the world in 2019. 02.15.2020. (In Russ.)]. Available at: https://ultramodern-home.ru/2020/02/samye-poseshhaemyestrany-mira-v-2019-godu/ (accessed 22.06.2021).

4. Pável A. Kuchínov. COVID-19: El nuevo orden mundial en el turismo. Iberoamérica. Moscow, 2021, núm. 1, pp. 28-50. Available at: https://iberoamericajournal.ru/sites/default/files/2021/1/kuchinov.pdf (accessed 15.08.2021).

5. Garcia Patricia J, Alarcón Alex, Bayer Angela, Buss Paulo, Guerra German, Ribeiro Helena, Rojas Karol, Rocío Saenz, Nelly Salgado de Snyder, Solimano Giorgio, Rubén Torres, Sebastián Tobar, Tuesca Rafael, Vargas Gilma and Atun Rifat. COVID-19 Response in Latin America. 2020 Nov. The American Journal of Tropical Medicine and Hygiene, Vol. 103: Issue 5. 2020. pp. 1765-1772. Available at: https://www.ajtmh.org/view/journals/tpmd/103/5/article-p1765.xml (accessed 07.06.2021).

6. Аналитическая записка. Последствия COVID-19 для Латинской Америки и Карибского бассейна. Июль 2020 года. ООН [Analytic note. Implications of COVID-19 for Latin America and the Caribbean. July 2020. UN. (In Russ.)]. Available at: https://www.un.org/sites/un2.un.org/files/the_impact_of_covid19_on_lac_russian.pdf (accessed 17.08.2021).

7. Концептуальная записка Генерального секретаря о туризме и COVID19. Август 2020 [Secretary General's Concept Note on Tourism and COVID-19. 
August 2020. (In Russ.)]. Available at: http://www.unic.ru/press/kontseptualnayazapiska-generalnogo-sekretarya-o-turizme-i-covid-19-avgust-2020-g (accessed 21.06.2021).

8. Pienknagura S., Roldós J., Werner A. Pandemic Persistence Clouds Latin America and Caribbean Recovery. October 22, 2020. Available at: https://blogs.imf.org/2020/10/22/pandemic-persistence-clouds-latin-americaand-caribbean-recovery/ (accessed 15.08.2021).

9. Financiación de la Atención de la Salud. Available at: https://www.paho.org/es/temas/financiacion-atencion-salud (accessed 18.08.2021).

10. Латинская Америка рискует стать главной жертвой коронавируса. Бразилия вышла на третье место в мире по числу зараженных, Аргентине грозит дефолт. 22.05.2020 [Latin America is at risk of becoming the main victim of the coronavirus. Brazil is in third place in the world in terms of the number of infected, Argentina is in danger of default. 05.22.2020. (In Russ.)]. Available at: https://meduza.io/feature/2020/05/22/latinskaya-amerika-riskuet-statglavnoy-zhertvoy-koronavirusa-braziliya-vyshla-na-tretie-mesto-po-chisluzarazhennyh-v-mire-argentine-grozit-defolt (accessed 02.08.2021).

11. COVID-19 in Latin America and the Caribbean: An overview of government responses to the crisis. Updated 11 November 2020. Available at: https://www.oecd.org/coronavirus/policy-responses/covid-19-in-latinamerica-and-the-caribbean-an-overview-of-government-responses-to-thecrisis-0a2dee41/ (accessed 07.08.2021).

12. Президент Мексики назвал локдауны плохим методом борьбы с COVID-19. 02.02.2021 [The Mexican President called lockdowns a bad method of dealing with COVID-19. 02.02.2021 (In Russ.)]. Available at: https://www.interfax.ru/world/739670 (accessed 24.07.2021).

13. Number of COVID-19 vaccine doses administered worldwide as of March 24, 2021, by country. Published by John Elflein, Mar 25, 2021. Available at: https://www.statista.com/statistics/1194934/number-of-covidvaccine-doses-administered-by-county-worldwide/ (accessed 28.07.2021).

14. Эпидемиологическая карта светофора [Epidemiological map of traffic lights (In Russ.)]. Available at: https://datos.covid19.conacyt.mx/\#SemaFE (accessed 23.07.2021).

15. World Bank. 2020. The Economy in the Time of Covid-19. LAC Semiannual Report/ World bank. April 12, 2020. Washington, 2020. Available at: https://openknowledge.worldbank.org/bitstream/handle/10986/33555/978146 4815706.pdf?sequence=10\&isAllowed=y (accessed 24.08.2021). 
16. Lew Alan A., Cheer Joseph M., Haywood Michael, Brouder Patrick \& Salazar Noel B. Visions of travel and tourism after the global COVID-19 transformation of 2020. June 2020 // Tourism Geographies. An International Journal of Tourism Space, Place and Environment. Available at: https://www.researchgate.net/publication/342025920_Visions_of_travel_and _tourism_after_the_global_COVID-19_transformation_of_2020 (accessed 03.08.2021).

17. Barbier E. B., Burgess J. C. Sustainability and Development after COVID-19. July 2020. Available at: https://www.researchgate.net/publication/342860379_Sustainability_and_De velopment_after_COVID-19 (accessed 24.07.2021).

18. Концептуальная записка: COVID-19 и перестройка сектора туризма. Август 2020 [Concept Note: COVID-19 and Restructuring the Tourism Sector. August 2020 (In Russ.)]. Available at: https://www.un.org/sites/un2.un.org/files/policy_brief_covid19_and_transforming_tourism_russian.pdf (accessed 21.07.2020).

19. Tourism Policy Responses to the coronavirus (COVID-19). Updated 2 June 2020. Available at: https://www.oecd.org/coronavirus/policyresponses/tourism-policy-responses-to-the-coronavirus-covid-19-6466aa20/ (accessed 14.08.2021).

20. WFH: how hotels are becoming offices for remote working. 06.08.2020. Available at: https://www.sleepermagazine.com/stories/originals/wfh-how-hotels-arebecoming-offices-for-remote-working/ (accessed 14.08.2021).

21. Cazres Jesus. The Impact of COVID-19 in Latin America and the Caribbean. Nov. 10, 2020. Available at: https://infomineo.com/impact-ofcovid-19-latin-america-caribbean/ (accessed 14.08.2021).

22. How COVID-19 has changed digital consumption in four of the largest Latin American countries. December 15, 2020. Available at: https://labsnews.com/en/articles/business/how-covid-19-has-changed-digitalconsumption-in-four-of-the-largest-latin-americancountries/?utm_campaign=LABS_EN\&utm_medium=email\&_hsmi=103165 642\&_hsenc=p2ANqtz-_j3-

1sgLQiZQpkIXZ2vCX43NgF7qGbuDYsrKdzoHnhwiVDLa43In9H-JM9CLQusWSeMZsIWILgcBYoEpNMmOXMD2hcw\&utm_content=1031641 91\&utm_source=hs_email (accessed 14.08.2021).

23. Number of e-commerce users in Latin America from 2017 to 2024 (in millions). Published by Stephanie Chevalier, Nov 24, 2020. Available at: https://www.statista.com/forecasts/251657/number-of-digital-buyers-in-latinamerica (accessed 16.08.2021). 
24. Number of confirmed cases of the novel coronavirus (COVID-19) in Latin America and the Caribbean as of April 26, 2021, by country. Published by Ana María Ríos, Apr 26, 2021. Available at: https://www.statista.com/statistics/1101643/latin-america-caribbeancoronavirus-cases/ (accessed 10.08.2021).

25. МВФ улучшил прогноз по ВВП стран Латинской Америки. 27 Июля 2021 [The IMF has improved its forecast for GDP in Latin America. July 27, 2021 (In Russ.)]. Available at: https://1prime.ru/state_regulation/20210727/834310427.html (accessed 16.08.2021).

26. Share of first-time buyers who plan to continue shopping online after the COVID-19 pandemic in selected Latin American countries in 2020. Published by Stephanie Chevalier. Jul 2, 2021. Available at: https://www.statista.com/statistics/1116643/share-consumers-shop-onlineafter-covid19-latin-america/ (accessed 16.08.2021).

27. Impact assessment of the COVID-19 outbreak on international tourism. Updated December 2020. Available at: https://www.unwto.org/impact-assessment-of-the-covid-19-outbreak-oninternational-tourism (accessed 14.08.2021).

28. Доклад Верховного комиссара Организации Объединенных Наций по правам человека «Воздействие пандемии коронавирусного заболевания (COVID-19) на осуществление прав человека во всем мире, в том числе передовая практика и вызывающие озабоченность области» [Report of the United Nations High Commissioner for Human Rights "Impact of the coronavirus disease (COVID-19) pandemic on the enjoyment of human rights around the world, including good practices and areas of concern" (In Russ.)]. Available at: https://reliefweb.int/sites/reliefweb.int/files/resources/A_HRC_46_19_R.pdf (accessed 23.07.2021).

29. Пололикашвили Зураб, Гурриа Анхель. Оказание поддержки и помощи в координации в интересах безопасного и устойчивого восстановления туризма. Мадрид, 5 октября 2020 [Pololikashvili Zurab, Gurria Anhel'. Okazanie podderzhki i pomoshchi v koordinacii v interesah bezopasnogo i ustojchivogo vosstanovleniya turizma. Madrid, 5 oktyabrya 2020 [Pololikashvili Zurab, Gurria Angel. Provide support and coordination assistance for safe and sustainable tourism recovery. Madrid, 5 October 2020 (In Russ.)]. Available at: https://webunwto.s3.eu-west1.amazonaws.com/s3fs-public/2020-10/201005-oecd-and-unwto-sg-ru.pdf (accessed 23.07.2021). 